\title{
Design et ethnographie
}

Comment les designers pratiquent les études de terrain

Lysianne Léchot Hirt, Nicolas Nova, Fabienne Kilchör et Sébastien Fasel

\section{(2) OpenEdition \\ Journals}

\author{
Édition électronique \\ URL : https://journals.openedition.org/tc/7560 \\ DOI : $10.4000 /$ tc. 7560 \\ ISBN : 0248-6016 \\ ISSN : 1952-420X \\ Éditeur \\ Éditions de l'EHESS
}

\section{Édition imprimée}

Date de publication : 24 décembre 2015

Pagination : 64-77

ISBN : 978-2-7132-2505-5

ISSN : 0248-6016

Référence électronique

Lysianne Léchot Hirt, Nicolas Nova, Fabienne Kilchör et Sébastien Fasel, « Design et ethnographie », Techniques \& Culture [En ligne], 64 | 2015, mis en ligne le 24 décembre 2018, consulté le 29 septembre 2022. URL : http://journals.openedition.org/tc/7560 ; DOI : https://doi.org/10.4000/tc.7560 


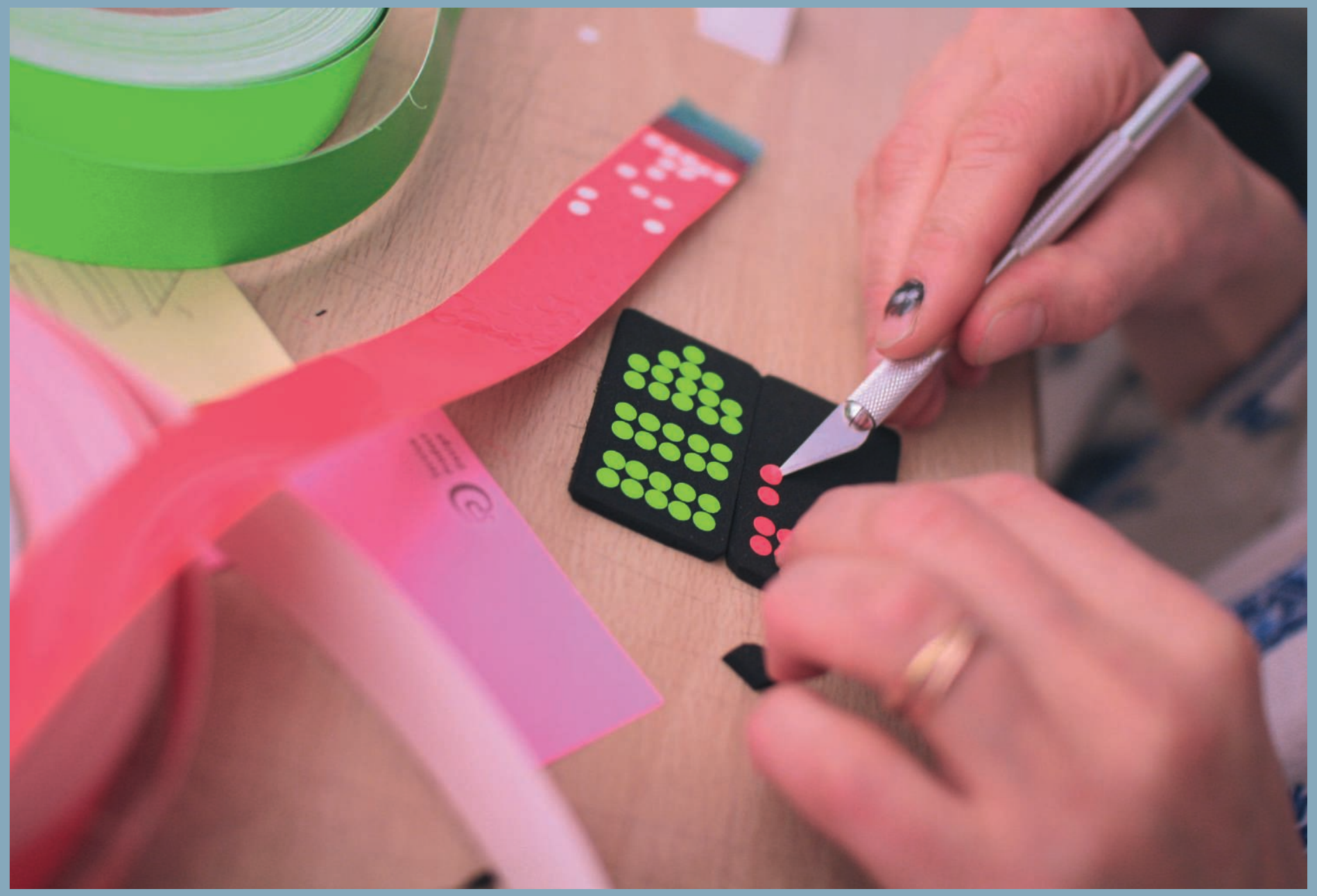




\section{Design et ethnographie} Comment les designers pratiquent les études de terrain

Comprendre les gens pour imaginer les objets envisageables pour eux: cet objectif utilitaire encadre les pratiques de design ethnography, apparues depuis les années 1980 dans le secteur des technologies de l'information et de la communication (Salvador 1999), puis dans le design industriel et de services (Norman 2013). Le terme et la pratique de design ethnography se sont imposés peu à peu en parallèle du user-centered design ou encore de la user research (Nova 2014). Il n'existe pas de définition unique de ce qui est désormais une expression-valise, recouvrant la plupart des pratiques d'enquêtes de terrain prises en charge dans le cadre de projets de design, présupposant que les connaissances acquises permettront le développement de produits plus pertinents pour leurs utilisateurs. Ce rapprochement du design et des sciences sociales a entraîné une appropriation de vocabulaires et d'outils issus des pratiques ethnographiques, non dénuée de biais empiriques et théoriques, en particulier lorsqu'il s'agit pour les designers d'expliquer comment ils utilisent les données, images et impressions produites au cours des recherches de terrain. Éclairer cette boîte noire est l'ambition de cet article, qui fait suite à une recherche entreprise au sein de la Haute-École d'Art et de Design de Genève en 2013-2014 1. Agissant au sein d'une école supérieure de design, nous avons notamment eu à cœur de produire pour nos étudiants des bases de savoirs pratiques solides et documentées, c'est pourquoi nous avons choisi de pratiquer notre recherche autour de la design ethnography à la fois en tant que chercheurs de terrain et designers.

Nous avons réalisé, pendant un an, vingt entretiens avec des designers sélectionnés en raison de leurs pratiques répétées de recherches de terrain, de leur bonne reconnaissance dans le milieu des technologies numériques (attestée par leur présence régulière dans des conférences, expositions ou salons professionnels), ainsi que de la diversité de leurs contextes de travail. Vivant en Suisse, Italie, France, Grande-Bretagne, Danemark et États-Unis, ils ont été contactés grâce à nos réseaux professionnels et de recherche. Les entretiens comportaient une phase de discussion libre 
Diagramme: cahier de suivi du projet réunissant tout document inspirant et lié au thème.

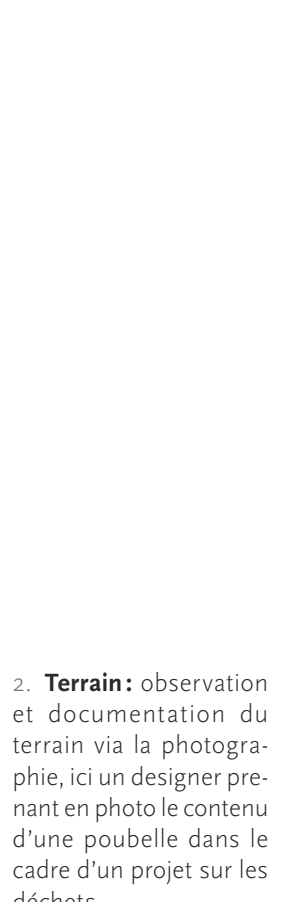

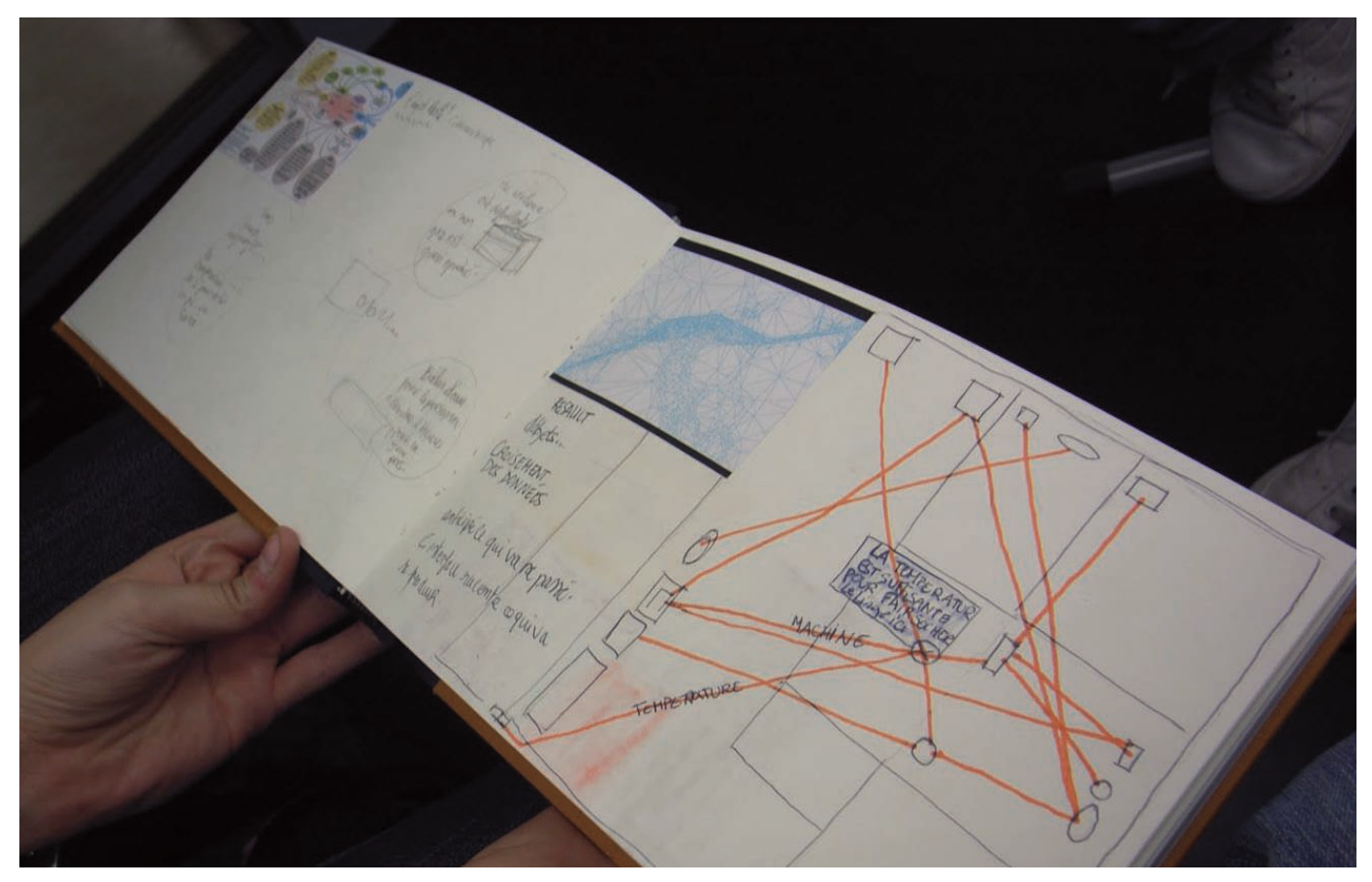

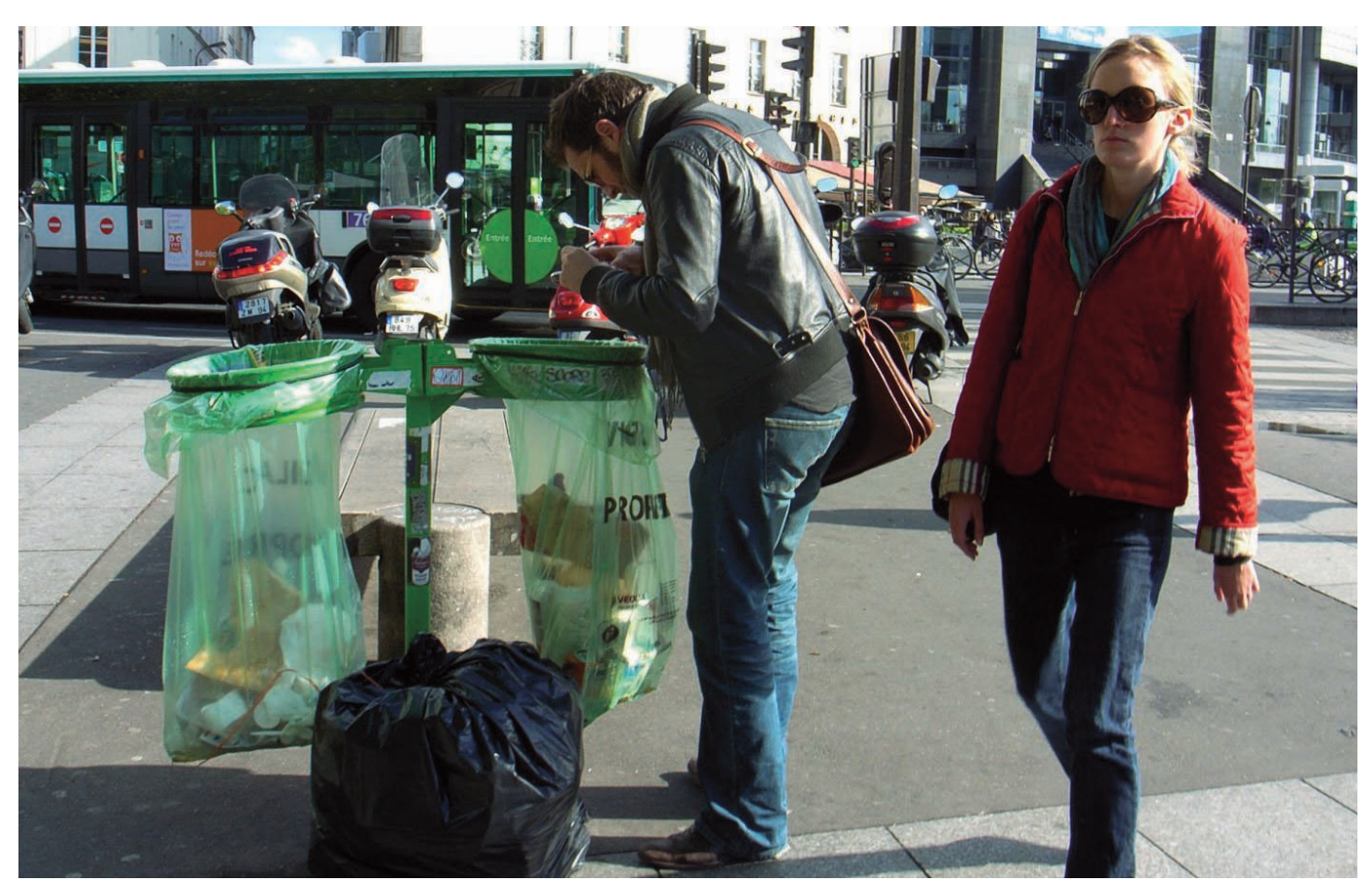


sur le rôle des usagers et de la recherche de terrain ainsi qu'une phase semi-directive à propos de leur carrière, du développement de leurs pratiques ethnographiques, de la description de projets récents avec un focus plus poussé sur les méthodes utilisées et leur influence sur la création. Nous avons documenté les productions de design utilisées durant le processus (esquisses, maquettes, objets, journaux d'usagers, séries d'images, créations de persona ou utilisateur-type) et avons observé deux sessions d'analyse collective (l'une juste après la réalisation des entretiens, l'autre en fin de projet lors d'un atelier de design organisé durant la conférence Lift à Genève) de données, de production d'esquisses et de prototypes. Nous avons organisé deux workshops avec des designers et des étudiants afin de tester les premières esquisses de modèles que notre étude a fait émerger.

Nous avions pour but de dégager des profils idéaux-typiques de designers-ethnographes en analysant les pratiques mais aussi de comprendre si et comment chaque profil engageait une manière spécifique de créer. Par ailleurs, deux designers graphiques spécialisés dans la visualisation de données ${ }^{2}$ faisaient partie de l'équipe de recherche et ont apporté leur point de vue de praticiens (designerly way of knowing: Cross 20063) en prenant notamment en charge la reformulation systématique de nos données qualitatives retranscrites sous forme de diagrammes, cartographies et matrices visuelles. Toutes nos analyses se sont fondées sur ces documents graphiques plutôt que sur une approche uniquement linguistique. Cette démarche inductive nous a menés à construire des catégories qui permettent de comprendre quelques aspects du bricolage intellectuel et pragmatique que constitue la pratique ethnographique des designers.

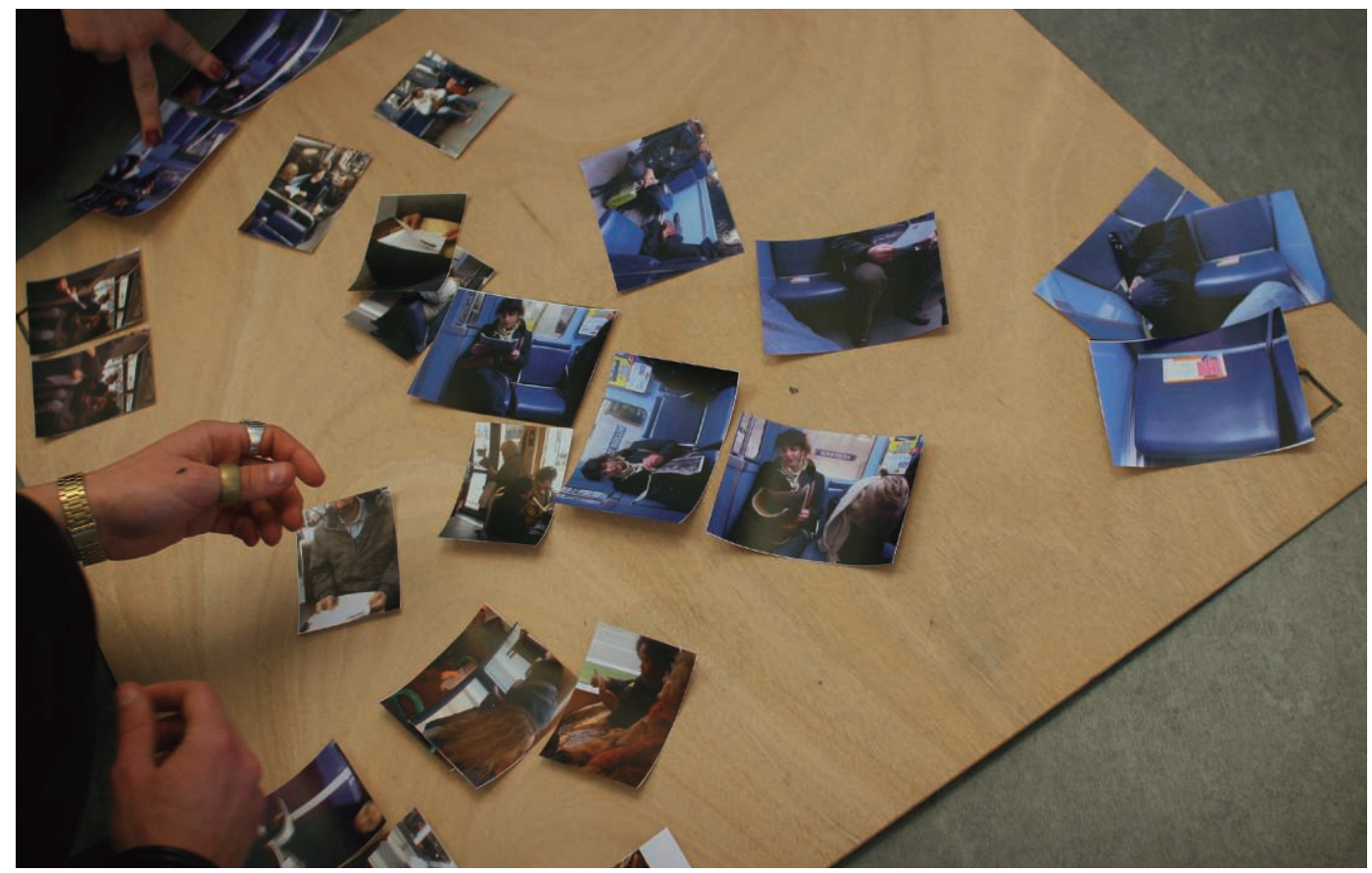

Classement: regroupement des photographies sur un tableau durant une phase d'analyse des données de terrain. 
4. Post-its employés pour résumer les observations et citations tirées des entretiens.

5. Parcours Utilisateur: représentation synthétique d'un parcours utilisateur ou d'un cours d'action sous forme de diagramme.
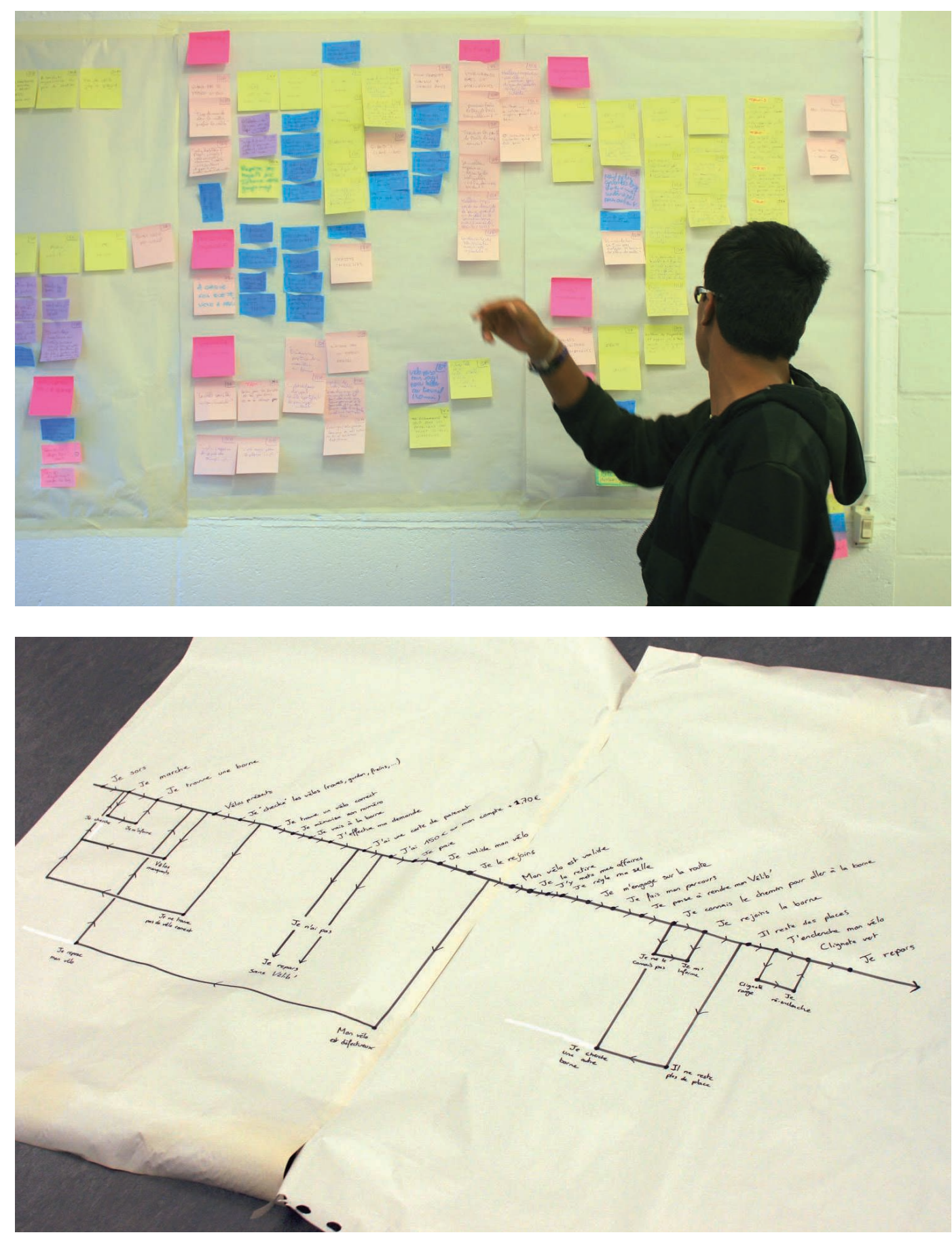


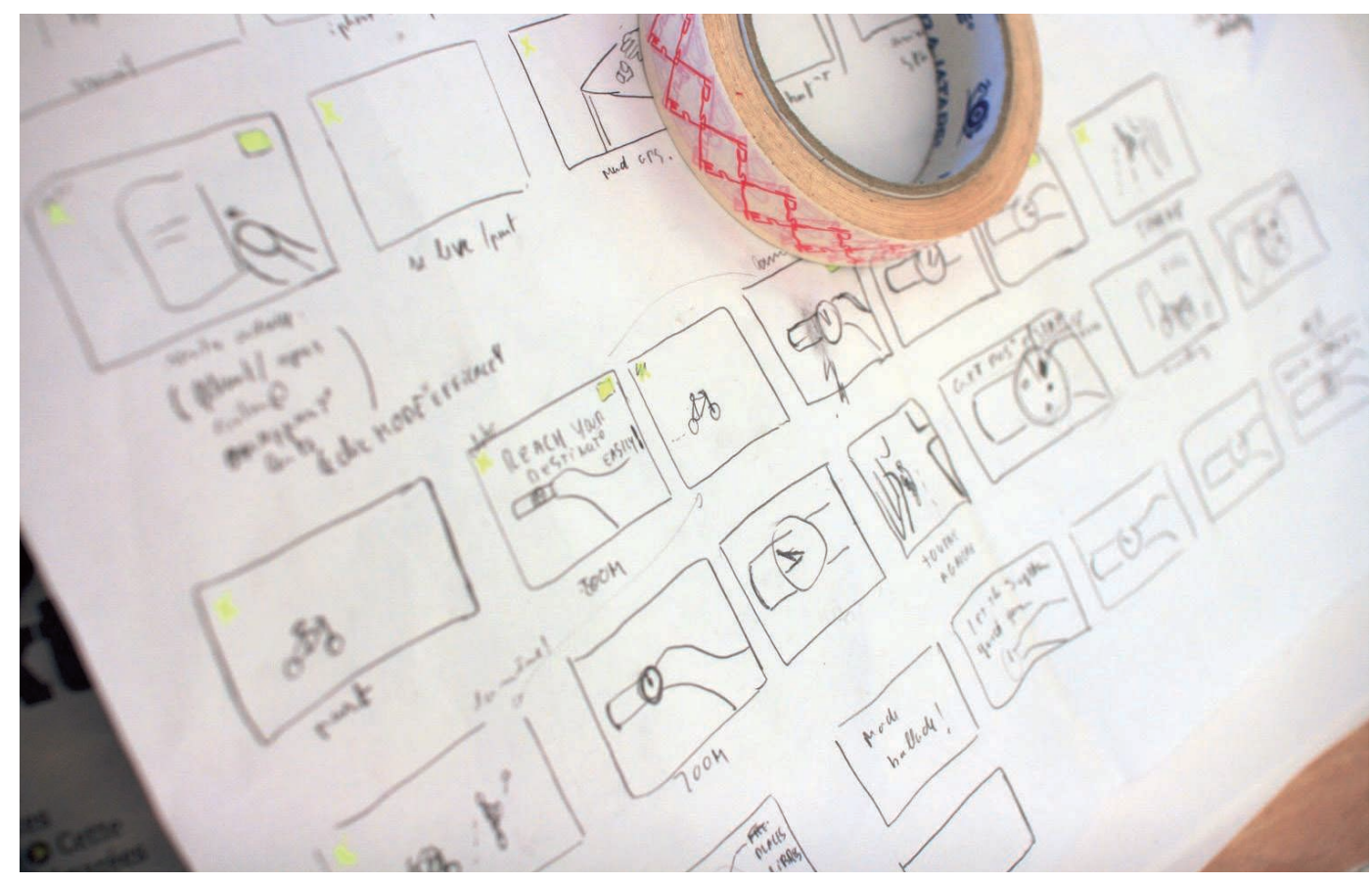

6. Storyboard: représentation schématique de l'utilisation d'un concept de design émergeant de l'étude ethnographique.

\section{Les designers enquêtent sur le terrain: manières de faire}

Trois motivations conduisent le designer à utiliser une démarche ethnographique: chercher l'inspiration en reformulant les problèmes posés par les clients; évaluer l'impact d'une idée; stimuler l'innovation en identifiant des désirs et des craintes émergentes. La mise en œuvre de la recherche de terrain comporte quatre phases qui se répètent ou se chevauchent. La réception du brief ${ }^{4}$ est suivie d'une préparation de la recherche puis d'une phase d'analyse qui précèdent enfin la production de propositions de design. Fréquemment, la préparation (consultation de projets existants, lecture de rapports, état de l'art) renouvelle la compréhension du brief, de même qu'elle fait émerger les véritables réflexions épistémologiques (que cherchons-nous à savoir? quel est vraiment le problème? comment choisir le terrain à explorer?). Le plus souvent, le designer hybride plusieurs outils ou en invente, pour pallier le manque de moyens ou

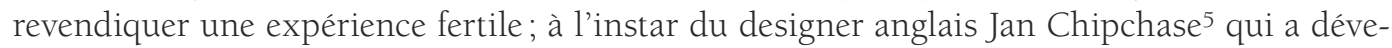
loppé sa méthode du «meanest motherfucker» («le pire connard») consistant à sélectionner des informateurs aussi peu idéaux que possible (Chipchase 2013) afin de le stimuler davantage dans la conception de produits originaux. Nous avons observé que les designers indépendants sont plus enclins à la créativité tandis que ceux liés à des entreprises utilisent des outils de marketing 
Prototypage rapide d'un concept de design avec des morceaux de carton. (sélection par catégorie d'âge, de genre, ou par catégorie socio-professionnelle). Cependant, tous sont prêts à répondre aux nécessités pratiques (accessibilité) par l'invention: ils privilégient les entretiens peu formalisés, les récits de pratiques complétés par des observations en tous genres, documentées en photo ou en vidéo (entretiens par Skype, usagers produisant eux-mêmes des photos ou des dessins, etc.). Tous les designers consultés développent ce qu'ils appellent des «stratégies de mixage» ou de «bricolage» de ces outils et revendiquent un rapport décontracté à la vérité empirique, se fiant souvent à une créativité émergeant sur le champ: «nous ne répétons jamais rien, nous modifions nos méthodes sur le moment», comme le résume l'un des designers interviewés.

Afin de partager la masse des données avec les clients, les designers produisent de nombreux résultats intermédiaires. Fiches, cartes postales, mood boards ${ }^{6}$, blogs abondent avant la phase d'analyse. Celle-ci est décrite comme un processus de construction de sens par raisonnement inductif en général implicite: en s’appuyant sur des données semblables (clustering), les designers font émerger des motifs ou des concepts. Avoir une vue d'ensemble, percevoir des catégories, identifier des exceptions, tout cela se fait de manière informelle (l'usage d'outils systématiques est rare). Pratiquement, l'analyse consiste en séances de travail collectif au cours desquelles on manipule les images, post-its, cartes postales, citations en les classant par affinités ou oppositions, ou, plus rarement, en suivant un modèle théorique ${ }^{7}$. À l'issue de cette phase, sont produits de nouveaux résultats visuels: schémas, mood boards, diaporamas, cartes, recueils illustrés, déjà

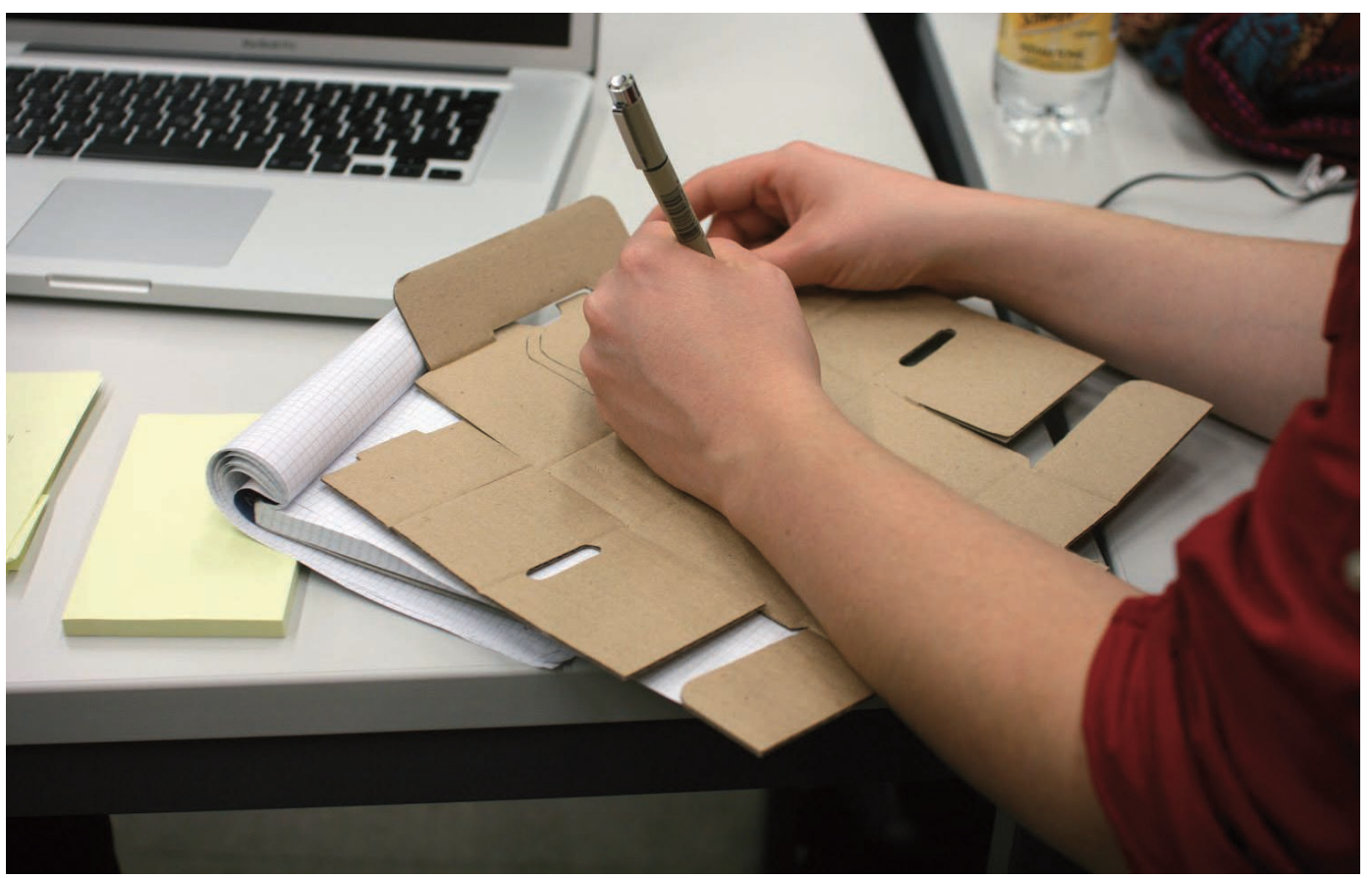


orientés vers leur applicabilité dans un futur projet de design. Ces résultats mettent en œuvre les compétences conceptuelles et plastiques des designers, ils sont décrits comme moyens de créer le dialogue avec les observations et doivent être dotés de qualités de lisibilité (économie de la forme et richesse des contenus) et d'ouverture (déclencheur de nouvelles idées).

\section{Andrea, Birdy, Charlie et les autres: cinq profils idéaux-typiques}

Notre propre méthode inductive, en particulier la traduction des données en schémas visuels, nous a conduits à construire cinq profils idéaux-typiques, mettant en évidence les différents positionnements épistémologiques et leurs conséquences en terme de choix de méthode d'enquête et de types de résultats de design produits (ou attendus) à l'issue du processus. Par exemple, Andrea, une designer indépendante travaillant pour des entreprises ou institutions de toutes natures, collabore avec des ethnologues quand le brief du client le lui enjoint et elle en attend une stimulation de ses inspirations. Elle considère le design comme une pratique destinée à satisfaire une demande et elle ne montre pas d'intérêt pour les discussions épistémologiques. Elle traite les données en privilégiant les motifs récurrents et peut se fier aussi

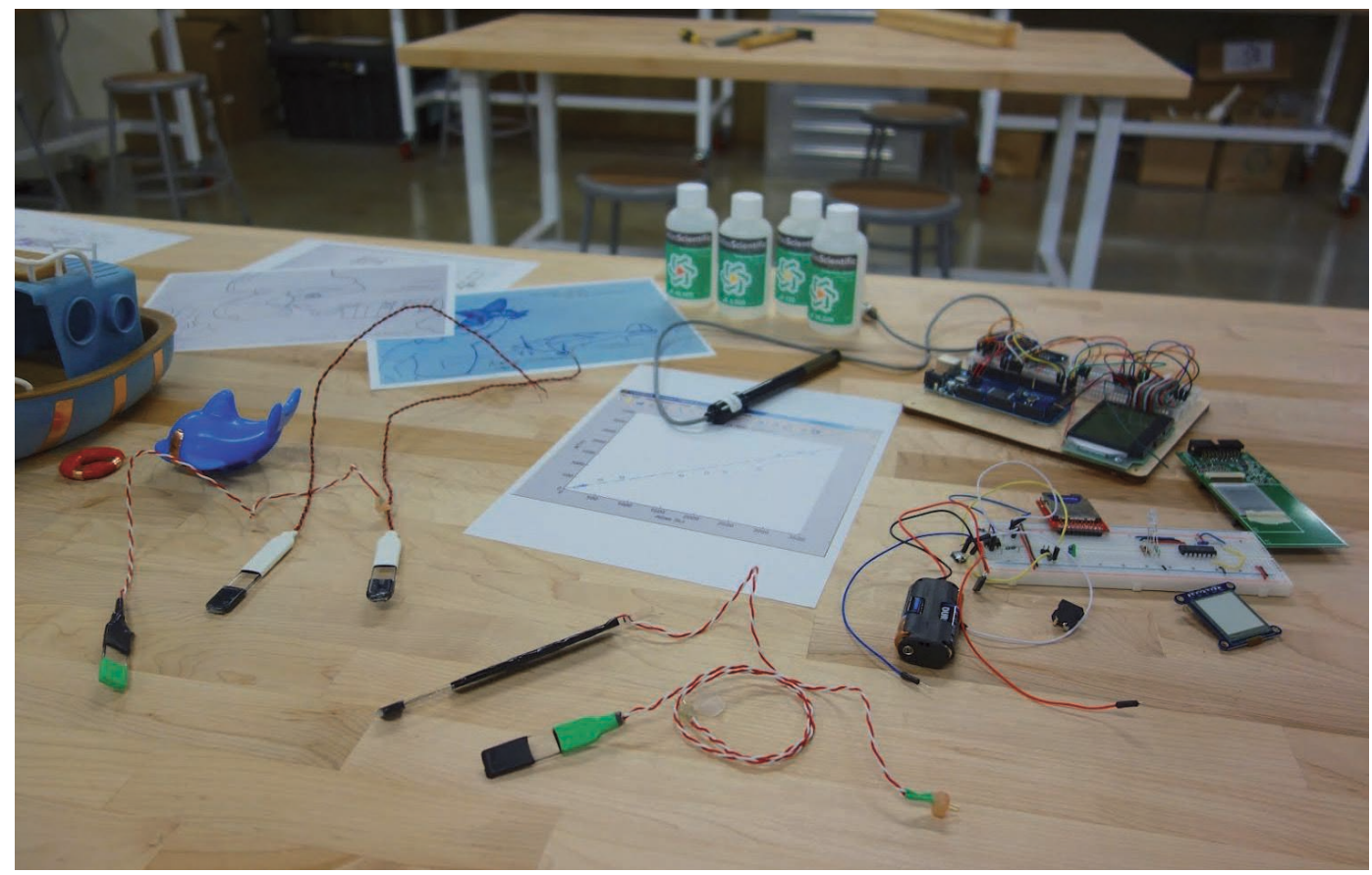

8. Prototypage rapide d'un concept de design avec un kit électronique. 
bien à des recherches ethnographiques sans en vérifier la qualité qu'à des statistiques élaborées rigoureusement. Ou bien Charlie, designer indépendant travaillant dans le secteur culturel, pour qui le design est certes destiné à apporter des solutions aux gens, mais aussi à permettre de développer sa propre expression personnelle. Il entreprend des recherches ethnographiques luimême par curiosité et pour stimuler son inspiration. Son rapport à la science est volontairement spéculatif, il joue avec la vérité empirique sans complexes et il est particulièrement intéressé par les dissonances des données, telles que les anecdotes. Les trois autres profils sont appelés Birdy, Dominique et Edwin. Birdy est une designer junior travaillant comme consultante pour des multinationales, elle est motivée par la résolution de problèmes et pour elle, le respect du brief est essentiel. Elle ne manifeste aucun intérêt pour la réflexion épistémologique et tend à répéter, dans ses enquêtes de terrain, les recettes ou méthodes dont on lui a garanti l'utilité et la scientificité. Dominique est employé dans une multinationale technologique et son approche est rigoureusement scientifique; il met en œuvre les méthodes éprouvées par son entreprise, et bien qu'il n’ait pratiquement jamais réfléchi à son positionnement épistémologique, il défend un point de vue positiviste, certain que sa démarche est la meilleure pour assurer le succès des produits qu'il conçoit. Edwin enfin représente le designer-chercheur employé dans une école ou un laboratoire universitaire; il favorise la rigueur scientifique et méthodologique et s'applique à formaliser ses recherches. Par ailleurs, il utilise l'environnement académique pour développer des méthodes innovantes et une réflexion critique sur la constitution des savoirs (attitude que nous qualifions d'épistémologie constructiviste), car pour lui ce n'est pas la réussite commerciale du produit ou la satisfaction du client qui comptent, mais la dimension originale de son travail, à même de le distinguer parmi ses pairs.

Nous notons que la corrélation entre les postures épistémologiques et les profils de designer est multilatérale. Quand un ou une designer n'a pas de connaissance ou d'avis sur les enjeux épistémologiques d'une enquête ethnographique, il peut assumer de travailler indifféremment dans tous les contextes, confiant dans le fait que cela l'aidera à trouver de meilleures solutions de design. À l'inverse, celui qui ne s'intéresse qu'aux données spéciales, construites par le designer-ethnographe, assume de facto une posture constructiviste sur le plan épistémologique, ce qui se traduit souvent par une critique des données statistiques ou un intérêt affirmé pour les questions méthodologiques, ce qui fut par exemple souligné dans un entretien avec le designer anglais James Auger, à propos de l'exploration de situations fictives (théâtrales) avec des acteurs en lieu et place d'enquêtes de terrain (Nova $\&$ al. 2014). La justification de cette méthode peu orthodoxe est soutenue théoriquement par des références philosophiques mais aussi pragmatiquement par sa fertilité pour les projets de design critique qui sont ceux du designer. Ce "Critical Design» correspond à une approche popularisée par Anthony Dunne et Fiona Raby, inventeurs du terme dans leurs publications Hertzian Tales (1999) puis Design Noir (2001). Il s'agit d'une démarche de création visant à susciter des débats sociaux à partir de la production et de l'exposition d'objets de design dérangeants ou indiquant des ruptures technologiques et sociétales. Ce design spéculatif vise davantage à la production d'idées et de débats qu'à la commercialisation de produits. 


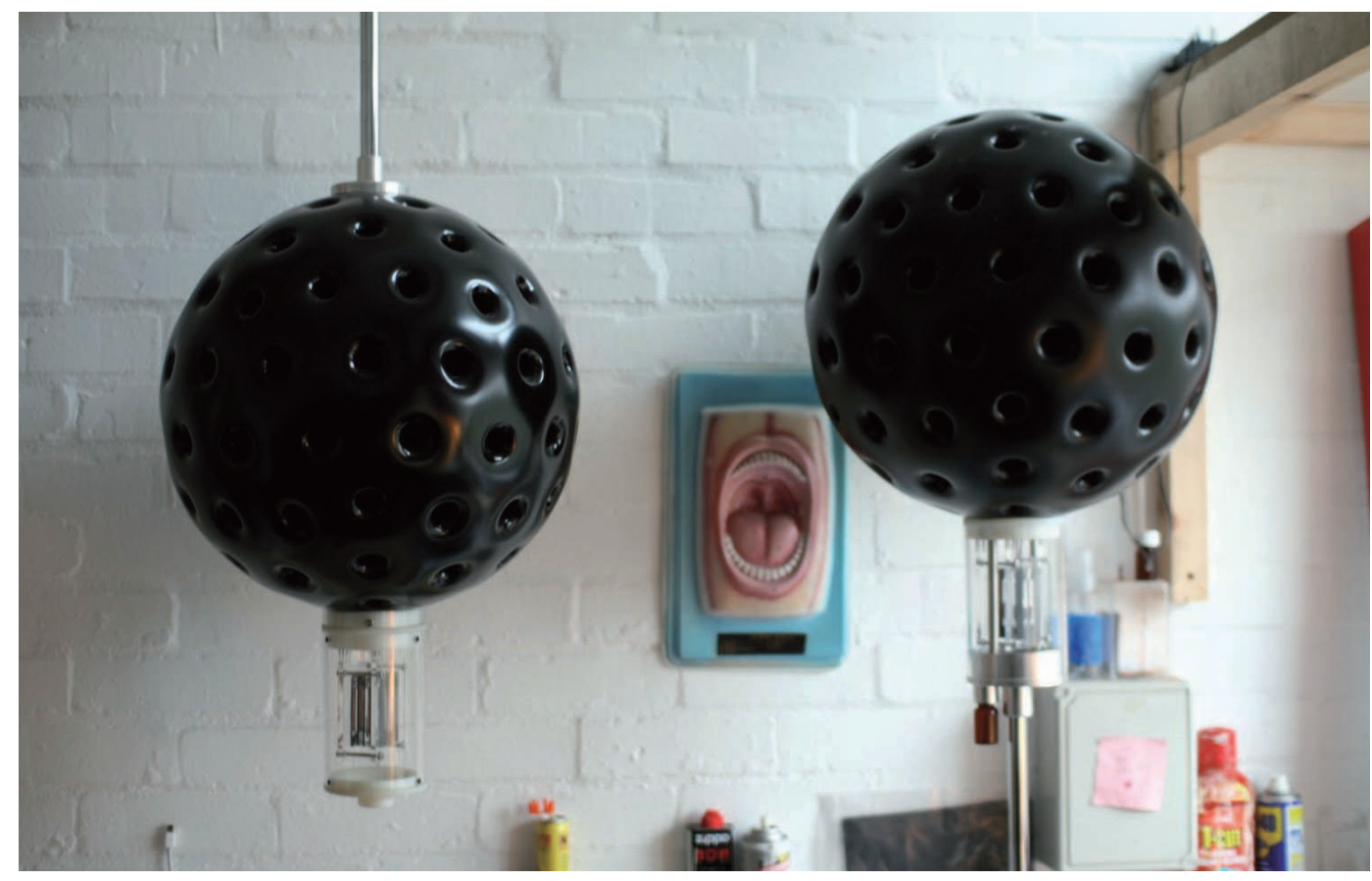

Il n'est en revanche pas évident d'affirmer qu'un positionnement épistémologique constructiviste, c'est-à-dire une propension à créer ses propres outils, méthodes et résultats, va de pair avec une vision du design que nous avons appelée « héroïque » (par opposition à scientifique ou pragmatique), centrée sur l'expression artistique du designer, héritier du génie romantique ${ }^{8}$, et à première vue le plus enclin à une inventivité de chaque instant. Un usage créatif des données, un rapport décontracté à l'épistémologie peuvent aussi servir un designer qui veut répondre aux besoins des usagers (pragmatique): «Je ne suis pas un designer motivé par l'ethnographie, mais je me soucie des gens» nous affirmait le designer allemand Fabian Hemmert (Nova \& al. 2014). C'est ce qui le pousse à engager une discussion avec des utilisateurs potentiels de ses prototypes de téléphones; par exemple, sur des objets changeant de forme suivant le nombre de messages reçus. Le matériau recueilli - des qualificatifs, des manières de décrire ces nouveaux objets, le vocabulaire proposé - lui permet ensuite de réorienter son projet. Et ce, sans aborder des enjeux d'acceptabilité ou d'attrait commercial comme aurait pu le faire un test marketing. Le cas de Sara Ljungblad nous éclaire aussi sur ce sujet, dans le cadre d'un projet de design orienté vers l'innovation (scientifique): la conception de robots innovants, en rupture avec la forme humanoïde. Celle-ci nous expliquait avoir étudié des propriétaires de reptiles domestiques (Nova \& al. 2014). La compréhension des motivations de ces personnes lui servant à imaginer comment des robots aux comportements singuliers pourraient permettre de sortir de formes robotiques très standardisées dans ce domaine.
Prototype finalisé d'un concept de design (Auger-Loizeau). 


\section{De l'observation du terrain au design: quatre gestes créatifs}

Cependant, les designers critiques et spéculatifs, en montrant la plus grande inventivité méthodologique, fertilisent tout particulièrement la manière dont l'observation (plus ou moins) ethnographique peut stimuler positivement la créativité. En effet, à partir des images, discours et impressions recueillies, ces designers enclenchent des gestes mentaux à même de produire des idées originales. Nous en relevons quatre, que nous synthétisons par des couples oppositionnels qui manifestent non une dialectique de type ou/ou, mais rendent sensible un éventail de possibles: inversion/translation, accélération/décélération, expansion/contraction, sophistication/simplification. Applicables aussi bien à la reformulation du brief qu'à la production de scénarios d'usage ou de prototypes, ces gestes mentaux sont ceux qui relient la conception plastique au donné empirique. Par exemple, une habitude observée dans l'usage d'un appareil ménager entraîne une idée pour le développement d'une «app» (application) pour tablette ou téléphone portable (translation du non-numérique au numérique), ou encore un geste répétitif et routinier est exagéré dans un scénario d'usage nouveau (décélération) - sortir son téléphone portable et le manipuler lorsqu'on est attablé avec des amis devant un verre pourrait donner lieu à un développement technologique qui permettrait de recharger la batterie dudit téléphone lorsqu'on l'agite (Nova 2012). L'important pour que cette traduction créative puisse avoir lieu est de conserver la complexité relativement floue des activités de terrain des designers-ethnographes. La relation à la vérité empirique est secondaire: ce qui prime, c'est que la production d'objets plastiques intermédiaires lors de l'analyse ne se différencie pas d'une production de design. La qualité et l'originalité formelles de ces outils de compréhension issus du design - par exemple le film Song of the Machine ${ }^{9}$ réalisé par le bureau de design londonien Superflux, dans lequel sont explorées des applications fictives d'une technologie émergente (Nova $\&$ al. 2014) - vont ici permettre une prise en compte des usagers, non pas dans le but de «faire de l'ethnographie», mais dans celui de voir le monde et de projeter ses transformations par le biais du design. En cela, la réappropriation par les designers des méthodes issues des sciences humaines démontre un art du bricolage particulièrement fertile. Pour Lévi-Strauss, bricoleur et ingénieur s'opposent (Lévi Strauss 1962) mais pour bon nombre de théoriciens du design il n'y a pas de différence fondamentale entre les démarches de l'artiste, du designer, de l'ingénieur ou du médecin (Simon 1981) qui tous observent le monde et les choses pour y déceler ce qui devrait être - et non simplement voir ce qui est. Fondamentalement créatrice, cette posture intellectuelle et existentielle s'autorise toujours des chemins de traverse. Alimentée par des références savantes - anthropologiques ou non - ou pratiquée de façon sauvage, la design ethnography, comme nous le précisions en introduction, est une pratique relativement récente dans l'histoire du design. Cependant les designers ont toujours eu à cœur d'orienter leur pratique vers les usagers, qu'il s'agisse des pionniers socialistes au xix siècle, comme William Morris donnant des conférences aux ouvriers; des designers industriels du Bauhaus dans la première moitié $\mathrm{du} \mathrm{xx}^{\mathrm{e}}$ siècle, soucieux des nouvelles formes de vie urbaine; ou plus près de nous des 
designers italiens des années 1960 et 1970, comme Enzo Mari décidant de partager ses dessins en libre accès afin que chacun puisse s'en emparer et construire ses propres meubles. Ou encore aujourd'hui, le graphiste Ruedi Baur défendant le «design civique ${ }^{10} »$ qui, à sa façon, continue d'affirmer la position centrale de la personne humaine et de ses besoins dans la pratique du design. L'approche ethnographique est l'une des briques du chantier du design, et en tant que telle une héritière et une continuatrice de toutes les inventions conceptuelles et méthodologiques qui l'ont construit.

\section{Notes}

1. Projet D-EX, Design Ethnography Exploration, soutenu par le Réseau de compétences Design et Arts visuels de la Haute École spécialisée de Suisse occidentale, HES-SO,

2. Fabienne Kilchör et Sébastien Fasel, créateurs du bureau Emphase à Lausanne: www.emphase.ch.

3. Designerly Ways of Knowing, publié par Cross en 2006 est un ouvrage majeur dans la production récente en théories du design. Son succès dans les communautés de la recherche en design a popularisé l'usage du qualificatif «designerly » dénotant une manière d'être, de faire ou de penser spécifique des designers.

4. Désignant usuellement le cahier des charges fourni au designer par le client (contexte du projet, description de ce qui est demandé avec une liste de contraintes), le «brief» peut également encadrer la demande de recherche.

5. Ancien chercheur en design chez Nokia et Frog Design, Jan Chipchase est l'une des figures majeures de cette approche de design ethnography, sujet sur lequel il a publié différents ouvrages méthodologiques.

6. Très utilisés dans certaines branches du design, le «tableau d'humeur» est un collage d'images, citations, échantillons de matériaux, qui résume l'univers d'inspiration.

7. Ces modèles, parfois académiques (théorie de l'activité), parfois pragmatiques (AEIOU: Activité

Environnement Interaction Objet Utilisateurs), fonctionnent comme des heuristiques dans la découverte des catégories au sein des données qualitatives disponibles.

8. L'époque romantique a popularisé la perception de l'artiste inspiré, ou même dominé, par des forces supérieures le poussant à créer, au détriment d'autres identités possibles, par exemple comme habile technicien ou comme penseur. Le «génie» est ainsi devenu un cliché dans l'opinion générale, qui admet que la création artistique est sa propre fin et que les comportements et discours des artistes n'ont pas à être justifiés. La sociologue Nathalie Heinich a bien montré les mécanismes de cette « exception artiste » et en a retracé la généalogie historique, notamment dans Lélite artiste. Excellence et singularité en régime démocratique (2005).

9. Résultant d'une collaboration entre une agence de design et un laboratoire de recherche, ce film de design spéculatif (http://superflux.in/work/ song-machine) décrit une technologie de prothèse rétinienne permettant une nouvelle forme de vision augmentée. Il témoigne des recherches actuelles au croisement des interfaces numériques et de la biotechnologie.

10. Voir le site www.civic-city.org.

\section{Les auteurs}

Sébastien Fasel est assistant de recherche HES à l'Institut de recherche en art et en design de la Haute École d'Art et de Design de Genève.

Fabienne Kilchör est designer et graphiste indépendante (diplômée en communication visuelle, Haute École d’Art et de Design de Genève). 
Lysianne Léchot Hirt est professeure HES à la Haute École d'Art et de Design de Genève; ses recherches actuelles portent sur la théorie et l'histoire du design.

Nicolas Nova est professeur à la Haute École d'Art et de Design de Genève et co-fondateur du Near Future Laboratory, une agence de prospective et d'innovation. Auteur de Futurs? La panne des imaginaires technologiques, il s'intéresse aux questions posées par les usages et le design des technologies de l'information et de la communication ainsi qu'aux cultures numériques.

\section{Iconographie}

Image d'ouverture. Prototypage rapide d'un concept de design avec des matériaux recyclés.

Crédits photographiques pour toutes les images: (C) Nicolas Nova.

\section{Références}

Chipchase, J. \& Steinhardt, S. 2013 Hidden in Plain Sight: How to Create Extraordinary Products for Tomorrow's Customer, New York: Harper Business.

Cross, N. 2006 Designerly Ways of Knowing, Berlin: Springer.

Dunne, A. 2009 [1999] Hertzian Tales. Electronic Products, Aesthetic Experience, and Critical Design, Cambridge: MIT Press.

Dunne, A. \& Raby, F. 2001 Design Noir. The Secret Life of Electronic Objects, Londres/Bâle: August/Birkhäuser.

Lévi-Strauss, C. 1962 La pensée sauvage, Paris: Plon.

Norman, D. 2013 [1988] The Design of Everyday Things. Revised and Expanded Edition, New York: Basic Books.

Nova, N., Léchot, L., Kilchör, F. \& Fasel, S. 2014 Beyond Design Ethnography. How Designers Practice Ethnographic Research, Berlin: SHS Publishers.

Nova, N., Miyake, K., Kwon, N. \& Chiu, W. 2012 Curious Rituals: gestural interactions in the digital everyday, Los Angeles: NFL Press. [En ligne] URL: https://curiousrituals.files.wordpress.com/2012/09/ curiousrituals-book.pdf Consulté le 29 octobre 2015.

Salvador, T., Bell, G. \& Anderson, K. 1999 « Design Ethnography», Design Management 10 (4) : 35-41.

Simon, H. 1981 The Sciences of the Artificial, Cambridge: MIT Press.

\section{Pour citer cet article}

Léchot Hirt, L., Nova, N., Kilchör, F. \& Fasel, S. 2015 « Design et ethnographie. Comment les designers pratiquent les études de terrain», TechniquesECulture no 64 «Essais de bricologie. Ethnologie de l'art et du design contemporains», p. 64-77. 
